Original Research Article

\title{
An observational study on the surgical antibiotic prophylaxis in the surgery ward of a tertiary care hospital
}

\author{
Aditi A. Kudchadkar*, Sushama A. Bhounsule
}

Department of Pharmacology,

Goa Medical College,

Bambolim Goa, India

Received: 06 May 2019

Accepted: 10 June 2019

\section{*Correspondence to:}

Dr. Aditi A. Kudchadkar,

Email: aditi101189@gmail.com

Copyright: (c) the author(s), publisher and licensee Medip Academy. This is an openaccess article distributed under the terms of the Creative Commons Attribution NonCommercial License, which permits unrestricted noncommercial use, distribution, and reproduction in any medium, provided the original work is properly cited.

\begin{abstract}
Background: Surgical site infection (SSI) is the most common postoperative complication and represents a significant burden in terms of patient morbidity, mortality and cost to health services around the world. Appropriate antibiotic prophylaxis helps in reducing the incidence of SSIs. Appropriate surgical prophylaxis is a multifactorial process that depends on proper case selection, antibiotic selection including dosing and route of administration and duration of therapy.

Methods: A prospective observational study was conducted over 3 months on 200 operated patients in surgery ward of a tertiary hospital. Patient details like demography, type of surgery performed and antibiotics prescribed pre and postsurgical procedure was collected and analysed using Microsoft excel.

Results: Out of 200 patients 32.5\% (65) were females and 67.5\% (135) were males. Total number of prophylactic antibiotics prescribed were 368 . The most commonly prescribed group of antibiotics was cephalosporins $(44.29 \%)$ followed by metronidazole $(26.5 \%)$ and ofloxacin $(9.48 \%)$. Among the cephalosporins the most commonly prescribed was cefuroxime in 82 patients $(50.3 \%$ ) and cefoperazone in 79 patients $(48.46 \%)$. SSI developed in 3 out of 200 patients (1.5\%).

Conclusions: There is an urgent requirement to promote rational antibiotic prescribing among surgeons. The need of the hour is developing and implementing national guidelines for surgical prophylaxis by a multidisciplinary group of experts.
\end{abstract}

Keywords: Antibiotic prescribing, Surgery, Surgical prophylaxis, Surgical site infection

\section{INTRODUCTION}

Surgical antimicrobial prophylaxis refers to the use of antibiotics for the prevention of surgical site infections and does not include pre-emptive use of antibiotics to treat early infections. ${ }^{1}$ The Centers for Disease Control and Prevention (CDC) has defined Surgical site infections (SSI) to standardize data collection for the National Nosocomial Infections Surveillance (NNIS) program. SSIs are classified into incisional SSIs, which can be superficial or deep, and organ/space SSIs, which affect the rest of the body other than the body wall layers. ${ }^{2}$
These classifications are defined as follows:

- Superficial incisional SSI - Infection involves only skin and subcutaneous tissue of incision

- Deep incisional SSI - Infection involves deep tissues, such as fascial and muscle layers; this also includes infection involving both superficial and deep incision sites and organ/space SSI draining through incision

- Organ/space SSI - Infection involves any part of the anatomy in organs and spaces other than the incision, which was opened or manipulated during operation. 
- $\quad$ SSI is the most common postoperative complication representing a significant burden in terms of patient morbidity and mortality thereby increasing the cost to health services around the world.

The incidence of SSIs can be effectively reduced by appropriate antibiotic prophylaxis. Inappropriate surgical prophylaxis continues to be a major problem nationally. Some studies suggest that nearly $30-50 \%$ of antibiotics used in hospitals are prescribed for surgical prophylaxis and $30-90 \%$ of this prophylaxis is inappropriate. ${ }^{3}$ In most of cases antibiotics were prescribed for durations longer than recommended in guidelines. ${ }^{4}$ It adds to the cost of medical care and unnecessarily exposes the patient to potential adverse effects. It also promotes the development and spread of bacterial resistance. ${ }^{5}$ Therefore appropriate surgical prophylaxis is a process that depends on multiple factors like proper case selection, antibiotic selection including dosing and route of administration and duration of therapy.

The aim of this study was to analyse the prescribing pattern of antibiotic prophylaxis in patients undergoing surgery and to observe the frequency of occurrence of surgical site infections in operated patients.

\section{METHODS}

The study was conducted over 3 months from $1^{\text {st }}$ November 2018 to $31^{\text {st }}$ January 2019 on operated patients in the surgery ward of Goa Medical College. Sample size was calculated to be 200 by estimation technique.

\section{Inclusion criteria}

- Patients of either sex, age 18 years or more

- Admitted in the ward for more than 24 hours for a surgical procedure

\section{Exclusion criteria}

- Admitted in the ward for less than 24 hours

- Patients not willing to participate.

After approval from the Institutional ethics committee, written informed consent was taken from the patients and the case records were analysed for patient details and the details of the antibiotics prescribed during the hospital stay.

Patient details included demographic details, clinical history, type of surgery performed and details of antibiotics prescribed pre and post-surgical procedure during the hospital stay. The data collected was analysed using Microsoft excel. The results are expressed as percentages. The results are depicted as graphs and charts wherever applicable. Rationality of preoperative antibiotic prophylaxis was evaluated as per guidelines of Scottish Intercollegiate Guideline Network (SIGN) ${ }^{6}$ and American Society of Health system Pharmacists (ASHP). ${ }^{7}$ These guidelines provide data to the practitioners for use of prophylactic antibiotics.

\section{Principles of surgical antibiotic prophylaxis ${ }^{1}$}

- Decide if prophylaxis is appropriate

- Determine the bacterial flora most likely to cause postoperative infection

- Choose an antibiotic, based on the steps above, with the narrowest antibacterial spectrum required

- Choose the less expensive drug if two drugs are otherwise of equal antibacterial spectrum, efficacy, toxicity, and ease of administration

- Administer dose at the right time

- Administer antibiotics for a short period (one dose if surgery of four hours duration or less)

- Avoid antibiotics likely to be of use in the treatment of serious sepsis

- Do not use antibiotic prophylaxis to overcome poor surgical technique

- Review antibiotic prophylaxis protocols regularly as both cost and hospital antibiotic resistance patterns may change.

\section{RESULTS}

\section{Demographics}

A total of 200 patients were included in the study. Out of this 200 patients studied $67.5 \%$ were males and $32.5 \%$ were females (Figure1).

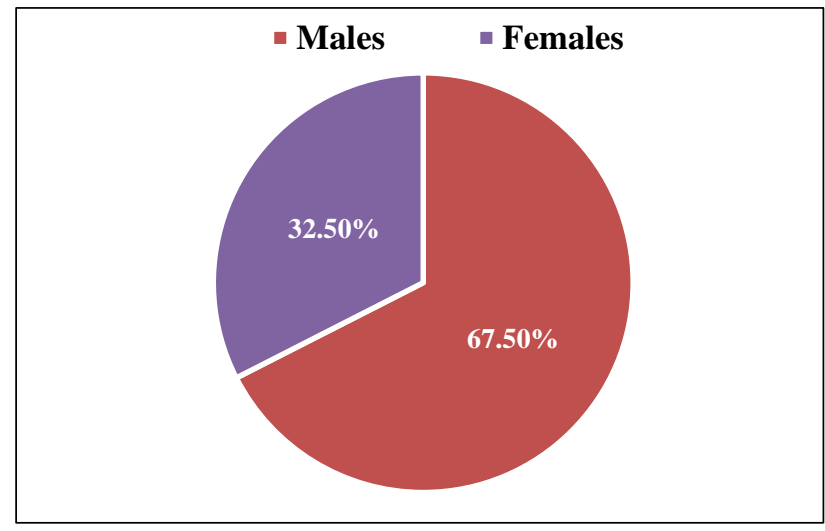

Most of the patients were in the age group of 41-50 years, with a mean age of 46.54 years. The majority $(62 \%)$ of patients had an elective procedure while $38 \%$ were operated on an emergency basis. The most common procedure performed was incision and drainage followed by appendicectomy and excision of cyst (Figure 2).

\section{Prescription analysis}

All the 200 patients received prophylactic antibiotics. All the antibiotics were given by intravenous route. The total number of prophylactic antibiotics prescribed in this study were 368 of which $69 \%$ were prescribed by generic name. 
Out of 200 patients $44 \%$ received a single antibiotic while $42.5 \%$ received a combination of 2 antibiotics and remaining received 3 or more antibiotics (Table 1).

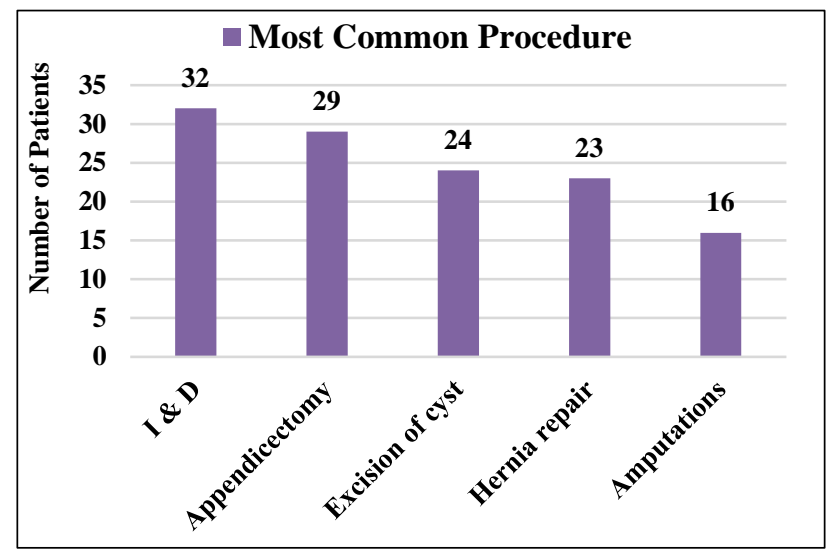

Figure 2: Type of procedure.

Table 1: Number of antibiotics prescribed for each patient.

\begin{tabular}{|ll|}
\hline Number of antibiotics & Number of patients \\
\hline 1 & $88(44 \%)$ \\
\hline 2 & $85(42.5 \%)$ \\
\hline 3 & $25(12.5 \%)$ \\
\hline 4 & $1(0.5 \%)$ \\
\hline
\end{tabular}

Among the total antibiotics prescribed the most common were cephalosporins (44.29\%) followed by metronidazole and the fluoroquinolone ofloxacin (Figure 3).

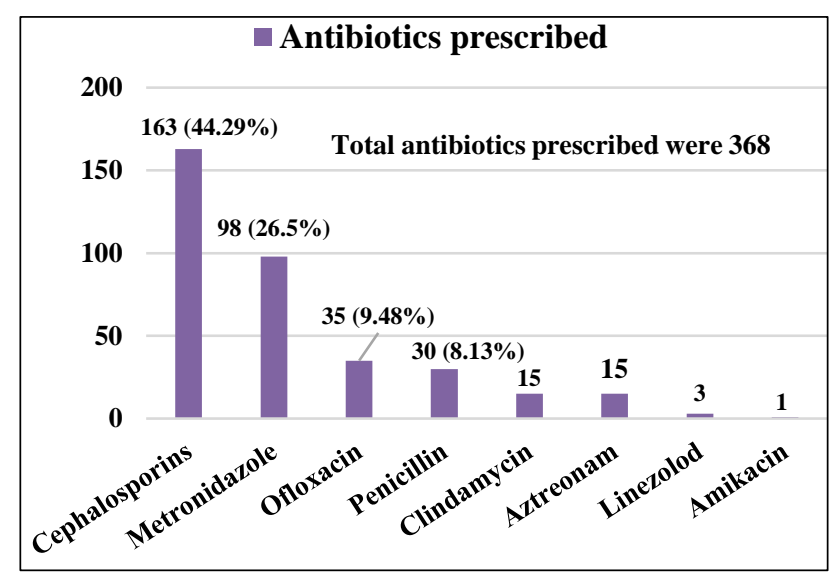

Figure 3: Antibiotics prescribed.

Among the cephalosporins the most commonly prescribed was a second generation cephalosporin cefuroxime in $50 \%$ of patients followed by the third generation cephalosporin cefoperazone. The first generation cephalosporin cefazolin was prescribed in only a single patient (Figure 4). A total of four different combinations of antimicrobials were used. The most common fixed dose combination was cefoperazone plus sulbactum followed by amoxicillin and clavulanic acid (Figure 5). Antipseudomonal antibiotic combination like piperacillin and tazobactam was given in one patient. Surgical site infection developed in 3 out of 200 patients $(1.5 \%)$. These patients complained mainly of purulent discharge from the site of infection. The patients received post-operative antibiotics for a mean duration of 6 days during their stay in hospital plus 5 days following discharge from ward. No adverse drug reaction was observed in this study.

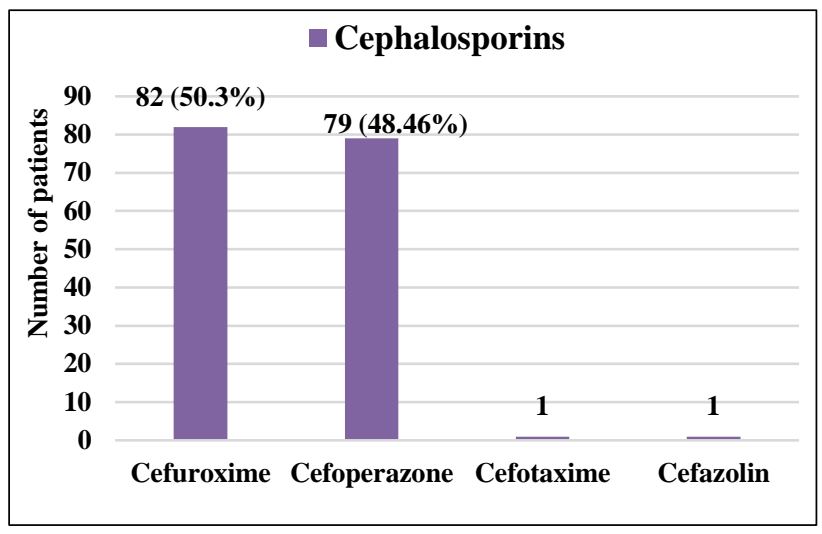

Figure 4: Cephalosporins prescribed.

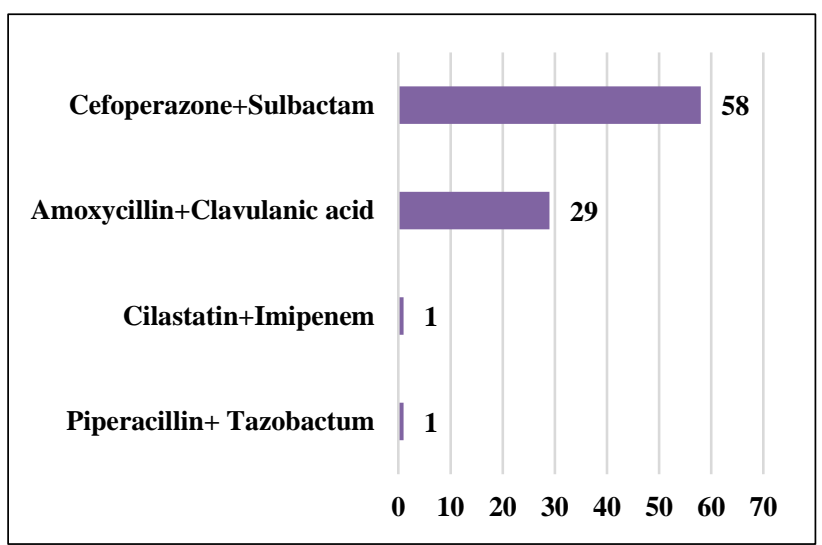

Figure 5: Combination of antibiotics prescribed.

\section{DISCUSSION}

The microbial load at the wound site is important in causing wound infection even with the usage of routine antibiotic prophylaxis. The protective function of skin is interrupted by skin incision, and microbial contamination is caused in spite of adequate skin preparation. Colonisation occurs with possibly pathogenic microorganism, and so any wound has the risk of infection.

The effectiveness of preoperative antibiotic prophylaxis is well established through this study. Drugs were prescribed by generic names in $69 \%$ of cases even though recent guidelines have been laid down by MCI to prescribe medicines only in generic names. The average number of antibiotics prescribed per patient was $1.67 .13 \%$ of patients received 3 or more antibiotics which is comparable to study conducted by Rehan et al $(17 \%){ }^{8}$ 
While the use of two or more antimicrobials in combination may have a certain rationale, their indiscriminate use can have numerous negative consequences. Possibly harmful aspects of such inappropriate antibiotic combinations include the development of bacterial resistance, super-infection, and the risks of toxicity, allergic reactions and increased cost of therapy. All the patients undergoing hernia repair and laparoscopic gall bladder surgeries received preoperative antibiotics while no such prophylaxis is recommended as per SIGN guidelines. ${ }^{6}$ This was also noted in studies by Rehan et al. ${ }^{8}$ In this study $21.7 \%$ of the patients received third generation cephalosporins .This number is similar to that observed by Kulkarni et al. ${ }^{9}(29 \%)$ and less than that observed by Rehan et al. ${ }^{8}$ (42\%). None of the patients received cefazolin(first generation) as recommended by SIGN guidelines. ${ }^{6}$

For surgical prophylaxis it is important to select an antibiotic with narrowest antibacterial spectrum to reduce the emergence of resistance and also because broad spectrum antibiotics may be required later if patient develops serious sepsis. Therefore, it is recommended that the use of third generation cephalosporins such as ceftriaxone and cefotaxime should be avoided in surgical prophylaxis. $^{1}$

Cefoperazone plus sulbactam was the most common combination prescribed in $29 \%$ patients in contrast to study conducted by Rehan et al. ${ }^{8}$ in which amoxicillin plus clavulanic acid was the most common FDC (12\%). The incidence of SSI was very low (1.5\%) similar to study Parulekar et al. ${ }^{10}$ This can be attributed to the fact that all the 200 patients received prophylactic antibiotics irrespective of whether it was appropriate or not. As far as duration of antimicrobial prophylaxis is concerned, one antibiotic dose is adequate for operations lasting 4 hours or less. Additional doses may be required in prolonged surgeries. ${ }^{1}$ Results from two large studies highlight the fact that single dose antibiotic prophylaxis is not associated with an increased rate of SSI when compared to multiple dose regimens. ${ }^{11,12}$

In this study, post-operative antibiotics were administered for a mean duration of 6 days during hospital stay and another 5 days following discharge, a period much longer than recommended. A similar duration of 6.4 days of antibiotic use has been reported in a study of taiwanese patients ${ }^{13}$ and an Indian study by Rehan et al. ${ }^{8}$ Persistence of tissue concentrations past the period of surgery increases toxicity and cost of therapy with no additional benefit. ${ }^{1}$

The present study was conducted on a small sample size (200) and for a short duration of time (3 months), hence it may not represent the total population. There have not been many studies done in India on this subject, therefore comparison of the results was limited.

\section{CONCLUSION}

Use of antimicrobials in all the cases was empirical based on operating surgeon's clinical experience. Increased use of antibiotics especially for a prolonged duration is a matter of concern. There is an urgent need to create awareness about rational antibiotic prescribing among surgeons. A multifaceted educational intervention involving a team effort of healthcare professionals can have a significant effect on effective antibiotic utilization and reducing the incidence of surgical site infections. Local guidelines seem more likely to be accepted and followed than those developed nationally. Development of similar antibiotic guidelines is essential to prevent emergence of resistant pathogens, to rationalize the use of antibiotics in the most cost-effective manner and for preventing the occurrence of hospital acquired infections..

\section{ACKNOWLEDGEMENTS}

Authors would like to thank staff of surgery department of Goa Medical College and Hospital.

Funding: No funding sources

Conflict of interest: None declared

Ethical approval: The study was approved by the Institutional Ethics Committee

\section{REFERENCES}

1. Munckhof W. Antibiotics for surgical prophylaxis. Australian Prescriber. 2005; 28(2):38-40.

2. Horan TC, Gaynes RP, Martone WJ, Jarvis WR, Emori TG. CDC definitions of nosocomial surgical site infections, 1992: a modification of CDC definitions of surgical wound infections. Infect Control Hosp Epidemiol. 1992;13(10):606-8.

3. Dettenkofer M, Forster D, Ebner W, Gastmeier P, Rüden H, Daschner F. The Practice of Perioperative Antibiotic Prophylaxis in Eight German Hospitals. Infection. 2002;30(3):164-7.

4. Kulkarni RA, Kochhar PH, Dargude VA, Rajadhyakshya SS, Thatte UM. Patterns of antimicrobial use by surgeons in India. Indian J Surg 2005;67(6):308-15.

5. Nichols R. Preventing Surgical Site Infections: A Surgeon's Perspective. Emerging Infectious Diseases. 2001;7(2):220-4.

6. Scottish Intercollegiate Guidelines Network. Antibiotic prophylaxis in surgery. Available at : http:// www.sign.ac.uk/pdf/sign104 Accessed 10th February 2019

7. ASHP Therapeutic Guidelines on Antimicrobial Prophylaxis in Surgery. Am J Health-System Pharmacy. 1999; 56(18):1839-88.

8. Rehan H, Kakkar A, Goel S. Surgical antibiotic prophylaxis in a tertiary care teaching hospital in India. Int J Infection Control. 2010; 66:12

9. Kulkarni RA, Kochhar PH, Dargude VA, Rajadhyakshya SS, Thatte UM. Patterns of 
antimicrobial use by surgeons in India. Indian J Surg 2005;67(6):308-15.

10. Parulekar L, Soman R, Singhal T, Rodrigues C, Dastur FD, Mehta A. How good is compliance with surgical antibiotic prophylaxis guidelines in a tertiary care private hospital in India? A prospective study. Indian J Surg. 2009;71(1):15-8.

11. Kanayama M, Hashimoto t, Shigenobu K, Osha F, Togawa D. Effective prevention of surgical site infection using a Centers for Disease Control and Prevention guideline-based antimicrobial prophylaxis in lumbar spine surgery. $J$ Neurosurg Spine. 2007;6(4):327-9.

12. Fonseca SN, Kunzle Sr, Junqueira MJ, Nascimento rt, de Andrade JI, Levin AS. Implementing 1-dose antibiotic prophylaxis for prevention of surgical site infection. Arch Surg. 2006;141(11):1109-3.

13. Chen YS, Liv YH, Kunin CM, Huang JK, Tsai CC. Use of prophylactic antibiotics in surgery at a Medical centre in southern taiwan. J Formos Med Assoc 2002;101(11):741-8.

Cite this article as: Kudchadkar AA, Bhounsule SA. An observational study on the surgical antibiotic prophylaxis in the surgery ward of a tertiary care hospital. Int J Basic Clin Pharmacol 2019;8:1647-51. 